\title{
Dirac quasinormal modes of two-dimensional charged dilatonic black holes
}

\author{
Ramón Bécar ${ }^{1, a}$, P. A. González ${ }^{2, b}$, Yerko Vásquez ${ }^{3, c}$ \\ ${ }^{1}$ Departamento de Ciencias Matemáticas y Físicas, Universidad Católica de Temuco, Montt 56, Casilla 15-D, Temuco, Chile \\ ${ }^{2}$ Facultad de Ingeniería, Universidad Diego Portales, Avenida Ejército Libertador 441, Casilla 298-V, Santiago, Chile \\ ${ }^{3}$ Departamento de Física, Facultad de Ciencias, Universidad de La Serena, Avenida Cisternas 1200, La Serena, Chile
}

Received: 16 May 2014 / Accepted: 11 June 2014 / Published online: 24 June 2014

(C) The Author(s) 2014. This article is published with open access at Springerlink.com

\begin{abstract}
We study charged fermionic perturbations in the background of two-dimensional charged dilatonic black holes, and we present the exact Dirac quasinormal modes. Also, we study the stability of these black holes under charged fermionic perturbations.
\end{abstract}

\section{Introduction}

The Einstein-Hilbert action is just a topological invariant in two spacetime dimensions (Gauss-Bonnet term) and, therefore, it is necessary to incorporate extra fields to add richness to the two-dimensional gravity models. In this sense, the dilatonic field plays the role of the extra fields, which naturally arises, for instance, in the compactifications from higher-dimensional models or from string theory. These theories also have black hole solutions which play an important role in revealing various aspects of the geometry of spacetime and the quantization of gravity, and also the physics related to string theory [1-3]. On the other hand, two-dimensional lowenergy string theory admits several black hole solutions. Furthermore, technical simplifications in two dimensions often lead to exact results, and it is hoped that this might help to address some conceptual problems posed by quantum gravity in higher dimensions. The exact solvability of twodimensional models of gravity has proven to be a useful tool for investigations into black hole thermodynamics [4-9]. It is hoped that such investigations provide a deeper understanding of key issues; including the microscopic origin of black hole entropy [10-12], and the end point of black hole evaporation via thermal radiation [13-15]. For an excellent review of dilaton gravity in two dimensions see [16]. Moreover, there

\footnotetext{
a e-mail: rbecar@uct.cl

b e-mail: pablo.gonzalez@udp.cl

c e-mail: yvasquez@userena.cl
}

is a growing interest in dilatonic black holes in the last few years, since it is believed that these black holes can shed some light on the solution of the fundamental problem of the microscopic origin of the Bekenstein-Hawking entropy. The area-entropy relation $S_{B H}=A / 4$ was obtained for a class of five-dimensional extremal black holes in Type II string theory using D-brane techniques [17]. In [2], the author derived the entropy for the two-dimensional black hole [3] by establishing the U-duality between the two-dimensional black hole and the five-dimensional one [2]. A similar work was carried out in [18] using a different sequence of duality transformations, this time in four dimensions, and leading to the same expressions for the entropy for two-dimensional black holes.

The particular motivation of this work is to calculate the quasinormal modes (QNMs) for charged fermionic field perturbations against the background of two-dimensional charged dilatonic black holes [3], and to study the stability of these black holes under charged fermionic perturbations. The QNMs and their quasinormal frequencies (QNFs) are an important property of black holes and have a long history [19-24]. The QNMs give information about the stability of black holes under matter fields that evolves perturbatively in the exterior region of them, without backreacting on the metric. Also, the QNMs determine how fast a thermal state in the boundary theory will reach thermal equilibrium according to the AdS/CFT correspondence [25], where the relaxation time of a thermal state of the boundary thermal theory is proportional to the inverse of the imaginary part of the QNFs of the dual gravity background [26]. In the context of black hole thermodynamics, the QNMs allow the quantum area spectrum of the black hole horizon to be studied, as well as the mass and the entropy spectrum. In this regard, Bekenstein [27] was the first to propose the idea that in quantum gravity the area of black hole horizon is quantized, leading to a discrete spectrum which is evenly spaced. Then Hod [28] conjectured that the asymptotic QNF is related to the 
quantized black hole area, by identifying the vibrational frequency with the real part of the QNFs. However, it is not universal for every black hole background. Then Kunstatter [29] proposed that the black hole spectrum can be obtained by imposing the Bohr-Sommerfeld quantization condition to an adiabatic invariant quantity involving the energy and the vibrational frequency. Furthermore, Maggiore [30] argued that in the large damping limit the identification of the vibrational frequency with the imaginary part of the QNF could lead to the Bekenstein universal bound. Then the consequences of these proposals were studied in several spacetimes. Besides, in [31-34] the authors discussed a connection between Hawking radiation and black hole quasinormal modes, which is important in the route to quantizing gravity, because one can naturally interpret black hole quasinormal modes in terms of quantum levels. The issues of the classical and quantum stability of two-dimensional and fivedimensional dilatonic black holes were treated for instance in [35-40], and it was shown that the absorption cross section vanishes at the low and high frequency limit for these black holes [41]. Also, the QNMs for charged dilatonic black holes were studied in [42] via semi-analytic and numerical methods.

This paper is organized as follows. In Sect. 2, we study charged fermionic perturbations in the background of twodimensional charged dilatonic black holes, and we calculate the exact QNMs. Finally, remarks are presented in Sect. 3.

\section{Charged fermionic perturbations of two-dimensional charged dilatonic black holes}

Maxwell gravity coupled to a dilatonic field $(\phi)$ can be described by the effective action [3]

$S=\frac{1}{2 \pi} \int d^{2} x \sqrt{-g} e^{-2 \phi}\left(R-4(\nabla \phi)^{2}-\lambda-\frac{1}{4} F_{\mu \nu} F^{\mu \nu}\right)$,

where $R$ is the Ricci scalar, $\lambda$ is the effective central charge, and $F_{\mu \nu}$ is the electromagnetic strength tensor. The equations of motion for the metric, gauge, and dilaton fields are given by

$R_{\mu \nu}-2 \nabla_{\mu} \nabla_{\nu} \phi-\frac{1}{2} F_{\mu \sigma} F_{\nu}^{\sigma}=0$,

$\nabla_{\nu}\left(e^{-2 \phi} F^{\mu \nu}\right)=0$

$R-4 \nabla_{\mu} \nabla^{\mu} \phi+4 \nabla_{\mu} \phi \nabla^{\mu} \phi-\lambda-\frac{1}{4} F_{\mu \nu} F^{\mu \nu}=0$.

The general static metric describing charged black holes in this theory can be written as

$d s^{2}=-f(r) d t^{2}+\frac{d r^{2}}{f(r)}$, where $f(r)=1-2 m e^{-Q r}+q^{2} e^{-2 Q r}, \phi=\phi_{0}-\frac{Q}{2} r$, and $F_{t r}=\sqrt{2} Q q e^{-Q r}$. The condition of asymptotic flatness for the spacetime requires $\lambda=-Q^{2}$. The free parameter $m$ is proportional to the black hole mass, and $q$ is proportional to the black hole electric charge. The horizons of the black hole are located at

$r_{ \pm}=\frac{1}{Q} \ln \left(m \pm \sqrt{m^{2}-q^{2}}\right) ;$

therefore, the condition $m^{2}-q^{2} \geqslant 0$ must be satisfied in order to have an event horizon at $r_{+}$. The change of coordinate $y=e^{-Q r}$ yields $f(y)=1-2 m y+q^{2} y^{2}$ where the spatial infinity is now located at $y=0$. This solution represents a well-known string-theoretic black hole [1-3]. The charged fermionic perturbations against the background of two-dimensional dilatonic black holes are governed by the Dirac equation in curved space,

$$
\left(\gamma^{\mu}\left(\nabla_{\mu}-i q^{\prime} A_{\mu}\right)+m^{\prime}\right) \psi=0,
$$

where $A_{\mu}$ is the electromagnetic potential, $q^{\prime}$ and $m^{\prime}$ are the electric charge and the mass of the fermionic field $\psi$, respectively, and the covariant derivative is defined as

$\nabla_{\mu}=\partial_{\mu}+\frac{1}{2} \omega_{\mu}^{a b} J_{a b}$

where $J_{a b}=\frac{1}{4}\left[\gamma_{a}, \gamma_{b}\right]$ are the generators of the Lorentz group. The gamma matrices in curved spacetime, $\gamma^{\mu}$, are defined by $\gamma^{\mu}=e_{a}^{\mu} \gamma^{a}$, where $\gamma^{a}$ are the gamma matrices in flat spacetime. In order to solve the Dirac equation, we use the diagonal vielbein

$e^{0}=\sqrt{f(r)} d t, \quad e^{1}=\frac{1}{\sqrt{f(r)}} d r$.

From the null torsion condition $d e^{a}+\omega_{b}^{a} \wedge e^{b}=0$, we obtain the spin connection

$\omega^{01}=\frac{f^{\prime}(r)}{2 \sqrt{f(r)}} e^{0}$.

Now, by using the following representation of the gamma matrices:

$\gamma^{0}=i \sigma^{2}, \quad \gamma^{1}=\sigma^{1}$

where $\sigma^{i}$ are the Pauli matrices, along with the following ansatz for the fermionic field:

$\psi=\frac{1}{f(r)^{1 / 4}} e^{-i \omega t}\left(\begin{array}{c}\psi_{1} \\ \psi_{2}\end{array}\right)$,

we obtain the following equations:

$$
\begin{aligned}
& \sqrt{f} \partial_{r} \psi_{1}+\frac{i \omega}{\sqrt{f}} \psi_{1}+\frac{\sqrt{2} i q q^{\prime}}{\sqrt{f}} e^{-Q r} \psi_{1}+m^{\prime} \psi_{2}=0 \\
& \sqrt{f} \partial_{r} \psi_{2}-\frac{i \omega}{\sqrt{f}} \psi_{2}-\frac{\sqrt{2} i q q^{\prime}}{\sqrt{f}} e^{-Q r} \psi_{2}+m^{\prime} \psi_{1}=0 .
\end{aligned}
$$


Decoupling the above system of equations, we obtain the following equation for $\psi_{1}$ :

$$
\begin{aligned}
& 2 f(r)^{2} \psi_{1}^{\prime \prime}(r)+f(r) f^{\prime}(r) \psi_{1}^{\prime}(r) \\
& +e^{-2 Q r}\left(4 q^{2} q^{\prime 2}+4 \sqrt{2} e^{Q r} q q^{\prime} \omega+2 e^{2 Q r} \omega^{2}\right. \\
& -2 e^{Q r}\left(m^{\prime 2} e^{Q r}+\sqrt{2} i q q^{\prime} Q\right) f(r) \\
& \left.-i e^{Q r}\left(\sqrt{2} q q^{\prime}+e^{Q r} \omega\right) f^{\prime}(r)\right) \psi_{1}(r)=0 .
\end{aligned}
$$

Now, making the change of variables $y=e^{-Q r}$, and after some algebraic manipulations, (12) becomes

$$
\begin{aligned}
& \psi_{1}^{\prime \prime}(y)+\left(\frac{1}{y}+\frac{1 / 2}{y-y_{+}}+\frac{1 / 2}{y-y_{-}}\right) \psi_{1}^{\prime}(y) \\
& +\left(\frac{A_{1}}{y}+\frac{A_{2}}{y-y_{+}}+\frac{A_{3}}{y-y_{-}}\right) \\
& \times \frac{1}{y\left(y-y_{+}\right)\left(y-y_{-}\right)} \psi_{1}(y)=0 .
\end{aligned}
$$

Here $y_{ \pm}$are the roots of $f(y)=1-2 m y+q^{2} y^{2}$, and they are given explicitly by

$y_{ \pm}=\frac{m \mp \sqrt{m^{2}-q^{2}}}{q^{2}}$,

and the constants $A_{1}, A_{2}$, and $A_{3}$ are defined by the following expressions:

$$
\begin{aligned}
A_{1} & =\frac{1}{q^{2} Q^{2}}\left(\omega^{2}-m^{\prime 2}\right), \\
A_{2} & =y_{+}\left(y_{+}-y_{-}\right) \\
& \times\left(\frac{1}{16}-\left(\frac{1}{4}-\frac{i \omega}{q^{2} Q y_{+}\left(y_{+}-y_{-}\right)}-\frac{\sqrt{2} i q^{\prime}}{q Q\left(y_{+}-y_{-}\right)}\right)^{2}\right),
\end{aligned}
$$

$$
\begin{aligned}
A_{3} & =-y_{-}\left(y_{+}-y_{-}\right) \\
& \times\left(\frac{1}{16}-\left(\frac{1}{4}+\frac{i \omega}{q^{2} Q y_{-}\left(y_{+}-y_{-}\right)}+\frac{\sqrt{2} i q^{\prime}}{q Q\left(y_{+}-y_{-}\right)}\right)^{2}\right) .
\end{aligned}
$$

Furthermore, performing another change of variable $z=\frac{y_{+}-y}{y_{+}}$, (13) reduces to

$$
\begin{gathered}
\psi_{1}^{\prime \prime}(z)+\left(\frac{1}{z-1}+\frac{1 / 2}{z}+\frac{1 / 2}{z-1+\frac{y_{-}}{y_{+}}}\right) \psi_{1}^{\prime}(z) \\
+\frac{1}{y_{+}^{2}}\left(\frac{A_{1}}{z-1}+\frac{A_{2}}{z}+\frac{A_{3}}{z-1+\frac{y_{-}}{y_{+}}}\right) \\
\times \frac{1}{z(z-1)\left(z-1+\frac{y_{-}}{y_{+}}\right)} \psi_{1}(z)=0 .
\end{gathered}
$$

We note that the above equation corresponds to the Riemann differential equation, whose general form is [43]

$$
\begin{aligned}
& \frac{d^{2} w}{d z^{2}}+\left(\frac{1-\alpha-\alpha^{\prime}}{z-r}+\frac{1-\beta-\beta^{\prime}}{z-s}+\frac{1-\gamma-\gamma^{\prime}}{z-t}\right) \frac{d w}{d z} \\
& +\left(\frac{\alpha \alpha^{\prime}(r-s)(r-t)}{z-r}+\frac{\beta \beta^{\prime}(s-t)(s-r)}{z-s}\right. \\
& \left.+\frac{\gamma \gamma^{\prime}(t-r)(t-s)}{z-t}\right) \frac{w}{(z-r)(z-s)(z-t)}=0
\end{aligned}
$$

where $r, s, t$ are the singular points, and the exponents $\alpha, \alpha^{\prime}, \beta, \beta^{\prime}, \gamma, \gamma^{\prime}$ are subject to the condition

$\alpha+\alpha^{\prime}+\beta+\beta^{\prime}+\gamma+\gamma^{\prime}=1$.

The complete solution of (19) is denoted by the symbol

$w=P\left\{\begin{array}{cccc}r & s & t & \\ \alpha & \beta & \gamma & z \\ \alpha^{\prime} & \beta^{\prime} & \gamma^{\prime} & \end{array}\right\}$,

where the $P$ symbol denotes the Riemann $P$ function, which can be reduced to the hypergeometric function through

$$
\begin{aligned}
w & =\left(\frac{z-r}{z-s}\right)^{\alpha}\left(\frac{z-t}{z-s}\right)^{\gamma} \\
& \times P\left\{\begin{array}{cccc}
0 & \infty & 1 & \\
0 & \alpha+\beta+\gamma & 0 & \frac{(z-r)(t-s)}{(z-s)(t-r)} \\
\alpha^{\prime}-\alpha & \alpha+\beta^{\prime}+\gamma & \gamma^{\prime}-\gamma &
\end{array}\right.
\end{aligned}
$$

where the $P$ function is now the Gauss hypergeometric function. So, considering (18) and (19) we can identify the regular singular points $r, s$, and $t$ as

$r=0, \quad s=1-\frac{y_{-}}{y_{+}}, t=1$

Therefore, the exponents are given by

$$
\begin{aligned}
& \alpha=-\frac{i \omega}{q^{2} Q y_{+}\left(y_{-}-y_{+}\right)}-\frac{\sqrt{2} i q^{\prime}}{q Q\left(y_{-}-y_{+}\right)}, \\
& \alpha^{\prime}=\frac{1}{2}+\frac{i \omega}{q^{2} Q y_{+}\left(y_{-}-y_{+}\right)}+\frac{\sqrt{2} i q^{\prime}}{q Q\left(y_{-}-y_{+}\right)}, \\
& \beta=\frac{i \omega}{q^{2} Q y_{-}\left(y_{-}-y_{+}\right)}+\frac{\sqrt{2} i q^{\prime}}{q Q\left(y_{-}-y_{+}\right)}, \\
& \beta^{\prime}=\frac{1}{2}-\frac{i \omega}{q^{2} Q y_{-}\left(y_{-}-y_{+}\right)}-\frac{\sqrt{2} i q^{\prime}}{q Q\left(y_{-}-y_{+}\right)}, \\
& \gamma=\frac{\sqrt{m^{\prime 2}-\omega^{2}}}{Q}, \quad \gamma^{\prime}=-\frac{\sqrt{m^{\prime 2}-\omega^{2}}}{Q},
\end{aligned}
$$


and the solution to (13) can be written as

$$
\begin{aligned}
& \psi_{1}(z)=C_{1}\left(\frac{z}{z-1+\frac{y_{-}}{y_{+}}}\right)^{\alpha}\left(\frac{z-1}{z-1+\frac{y_{-}}{y_{+}}}\right)^{\gamma} \\
& \quad{ }_{2} F_{1}\left(a, b, c, \frac{\frac{y_{-}}{y_{+}} z}{\left.z-1+\frac{y_{-}}{y_{+}}\right)}\right) \\
& +C_{2}\left(\frac{z}{z-1+\frac{y_{-}}{y_{+}}}\right)^{\alpha^{\prime}}\left(\frac{z-1}{z-1+\frac{y_{-}}{y_{+}}}\right)^{\gamma} \\
& \quad \times{ }_{2} F_{1}\left(a-c+1, b-c+1,2-c, \frac{y_{-}}{\left.z-1+\frac{y_{-}}{y_{+}}\right)}\right),
\end{aligned}
$$

where we have defined the constants $a, b$, and $c$ as

$$
\begin{aligned}
& a=\alpha+\beta+\gamma, \\
& b=\alpha+\beta^{\prime}+\gamma, \\
& c=1+\alpha-\alpha^{\prime} .
\end{aligned}
$$

In the near horizon limit, the above expression behaves as

$\psi_{1}(z \rightarrow 0)=\widehat{C}_{1} z^{\alpha}+\widehat{C}_{2} z^{\alpha^{\prime}}$,

where $\widehat{C}_{1}$ and $\widehat{C}_{2}$ are constants. Now, we impose the boundary condition that classically nothing can escape from the event horizon, therefore we must take $C_{2}=0$ in order to have only ingoing waves at the horizon. So, the solution simplifies to

$$
\begin{aligned}
\psi_{1}(z)= & C_{1}\left(\frac{z}{z-1+\frac{y_{-}}{y_{+}}}\right)^{\alpha}\left(\frac{z-1}{z-1+\frac{y_{-}}{y_{+}}}\right)^{\gamma} \\
& \times{ }_{2} F_{1}\left(a, b, c, \frac{\frac{y_{-}}{y_{+}} z}{\left.z-1+\frac{y_{-}}{y_{+}}\right)}\right) .
\end{aligned}
$$

Now, we implement boundary conditions at spatial infinity $z \rightarrow 1$. In order to do this, we employ the Kummer relations [43]

$$
\begin{aligned}
& { }_{2} F_{1}(a, b, c ; z)=\frac{\Gamma(c) \Gamma(c-a-b)}{\Gamma(c-a) \Gamma(c-b)} 2 \\
& \quad \times F_{1}(a, b, a+b-c ; 1-z) \\
& +(1-z)^{c-a-b} \frac{\Gamma(c) \Gamma(a+b-c)}{\Gamma(a) \Gamma(b)} 2 \\
& \quad \times F_{1}(c-a, c-b, c-a-b+1 ; 1-z),
\end{aligned}
$$

which allow us to write the solution as

$$
\begin{aligned}
& \psi_{1}(z)=C_{1}\left(\frac{z}{z-1+\frac{y_{-}}{y_{+}}}\right)^{\alpha}\left(\frac{z-1}{z-1+\frac{y_{-}}{y_{+}}}\right)^{\gamma} \\
& \times \frac{\Gamma(c) \Gamma(c-a-b)}{\Gamma(c-a) \Gamma(c-b)}{ }_{2} F_{1} \\
& \times\left(a, b, a+b-c, 1-\frac{\frac{y_{-}}{y_{+}}}{\left.z-1+\frac{y_{-}}{y_{+}}\right)}\right)
\end{aligned}
$$

$$
\begin{aligned}
& +C_{1}\left(1-\frac{y_{-}}{y_{+}}\right)^{\gamma^{\prime}-\gamma}\left(\frac{z}{z-1+\frac{y_{-}}{y_{+}}}\right)^{\alpha}\left(\frac{z-1}{z-1+\frac{y_{-}}{y_{+}}}\right)^{\gamma^{\prime}} \\
& \times \frac{\Gamma(c) \Gamma(a+b-c)}{\Gamma(a) \Gamma(b)} \\
& \times{ }_{2} F_{1}\left(c-a, c-b, c-a-b+1,1-\frac{\frac{y_{-}}{y_{+}} z}{\left.z-1+\frac{y_{-}}{y_{+}}\right)}\right) .
\end{aligned}
$$

In the limit $z \rightarrow 1$, the above expression becomes

$$
\begin{aligned}
& \psi_{1}(z \rightarrow 1)=\widetilde{C}_{1}(1-z)^{\gamma} \frac{\Gamma(c) \Gamma(c-a-b)}{\Gamma(c-a) \Gamma(c-b)} \\
& +\widetilde{C}_{1}\left(1-\frac{y_{-}}{y_{+}}\right)^{\gamma^{\prime}-\gamma}(1-z)^{\gamma^{\prime}} \frac{\Gamma(c) \Gamma(a+b-c)}{\Gamma(a) \Gamma(b)},
\end{aligned}
$$

where $\widetilde{C}_{1}$ is a constant. So, in order to have only outgoing waves at spatial infinity $z=1$, we must impose $c-a=$ $-n$ or $c-b=-n$. These conditions yield the following sets of quasinormal frequencies for two-dimensional charged dilatonic black holes:

$$
\begin{aligned}
& \omega_{1}=-\frac{\sqrt{2} m q q^{\prime}}{q^{2}}-i \frac{m Q \sqrt{m^{2}-q^{2}}}{2 q^{2}}(1+2 n)+i \frac{\sqrt{m^{2}-q^{2}}}{2 q^{2}} \\
& \times \sqrt{Q^{2}\left(m^{2}-q^{2}\right)(1+2 n)^{2}+4 q^{2}\left(m^{\prime 2}-2 q^{\prime 2}\right)-4 \sqrt{2} i q q^{\prime} Q \sqrt{m^{2}-q^{2}}(1+2 n)}
\end{aligned}
$$

and

$$
\omega_{2}=-i \frac{n Q}{2}+i \frac{m^{\prime 2}}{2 n Q},
$$

where $n=0,1,2, \ldots$ for the first set and $n=1,2, \ldots$ for the second set of frequencies. Depending on the value of the fermionic field mass $m^{\prime}$, some frequencies can have positive imaginary part, and therefore the black holes can be unstable. In order to separate the real part from the imaginary part for the first set of frequencies, we define

$$
\begin{aligned}
Z= & Q^{2}\left(m^{2}-q^{2}\right)(1+2 n)^{2}+4 q^{2}\left(m^{\prime 2}-2 q^{\prime 2}\right) \\
& -4 \sqrt{2} i q q^{\prime} Q \sqrt{m^{2}-q^{2}}(1+2 n)
\end{aligned}
$$

therefore, the first set of frequencies can be written as

$$
\begin{aligned}
\omega_{1}= & -\frac{\sqrt{2} m q q^{\prime}}{q^{2}}+\operatorname{sgn}\left(q q^{\prime}\right) \frac{\sqrt{m^{2}-q^{2}}}{2 q^{2}} \sqrt{\frac{|Z|-R e(Z)}{2}} \\
& -i \frac{\sqrt{m^{2}-q^{2}}}{2 q^{2}}\left(m Q(1+2 n)-\sqrt{\frac{|Z|+R e(Z)}{2}}\right),
\end{aligned}
$$

where $\operatorname{sgn}\left(q q^{\prime}\right)$ gives the sign of $q q^{\prime}$. Therefore, the imaginary part of the first set of frequencies is negative for

$m^{\prime 2}<\frac{m^{2} Q^{2}(1+2 n)^{2}+8 q^{2} q^{\prime 2}}{4 m^{2}}$. 
So, if the above inequality is satisfied, the two-dimensional charged dilatonic black holes are stable under charged fermionic perturbations. Note that in the case $q^{\prime} \neq 0$ the QNFs acquire a real part. Analogously, for $m^{\prime 2}<n^{2} Q^{2}$, the imaginary part of the second set of frequencies is negative, which guarantees the stability. Remarkably, this second set is the same as the QNFs for two-dimensional uncharged dilatonic black holes $[38,40]$. In a similar way, the QNFs associated to $\psi_{2}$ can be obtained, and we note that $\psi_{2}$ satisfies a similar equation to $\psi_{1}$ but making the changes $q^{\prime} \rightarrow-q^{\prime}$ and $\omega \rightarrow-\omega$. For $\psi_{2}$, and, following an analogous procedure, we find two additional sets of quasinormal frequencies. One of them is the same set of quasinormal frequencies as given in Eq. (37) and the other set is given by

$\omega_{3}=-i \frac{(n+1) Q}{2}+i \frac{m^{\prime 2}}{2(n+1) Q}$,

where $n=0,1,2, \ldots$ In this case, the imaginary part is negative when the fermionic field mass satisfies the inequality $m^{\prime 2}<(n+1)^{2} Q^{2}$.

\section{Final remarks}

In this work we have computed analytically the QNMs of charged fermionic perturbations for two-dimensional charged dilatonic black holes and we analyzed the stability of these black holes under fermionic perturbations. The fermionic fields are solutions of the Dirac equation, which can be reduced to the Riemann differential equation, as in $[44,45]$, and we have shown that there are two sets of quasinormal frequencies for each component of the fermionic field, where the second set, (39), is the same as that in the uncharged case $[38,40]$. On the other hand, the quasinormal frequencies of the first set are given by a real part, when $q^{\prime} \neq 0$, and an imaginary part, which can be negative if the square fermionic field mass satisfies (38).

Acknowledgments This work was funded by Comisión Nacional de Investigación Científica y Tecnológica through FONDECYT Grant 11121148 (Y.V.).

Open Access This article is distributed under the terms of the Creative Commons Attribution License which permits any use, distribution, and reproduction in any medium, provided the original author(s) and the source are credited.

Funded by $\mathrm{SCOAP}^{3}$ / License Version CC BY 4.0.

\section{References}

1. E. Witten, Phys. Rev. D 44, 314 (1991)

2. E. Teo, Phys. Lett. B 430, 57 (1998)

3. M.D. McGuigan, C.R. Nappi, S.A. Yost, Nucl. Phys. B 375, 421 (1992)

4. J.P.S. Lemos, Phys. Rev. D 54, 6206 (1996) gr-qc/9608016

5. D. Youm, Phys. Rev. D 61, 044013 (2000). hep-th/9910244

6. J.L. Davis, L.A. Pando Zayas, D. Vaman, JHEP 0403, 007 (2004) hep-th/0402152
7. D. Grumiller, R. McNees, JHEP 0704, 074 (2007). hep-th/0703230 [HEP-TH]

8. H. Quevedo, A. Sanchez, Phys. Rev. D 79, 087504 (2009). arXiv:0902.4488 [gr-qc]

9. A. Belhaj, M. Chabab, H. El Moumni, M.B. Sedra, A. Segui, Int. J. Geom. Methods Mod. Phys. 11(5), 1450047 (2014). arXiv:1311.2801 [hep-th]

10. R.C. Myers, Phys. Rev. D 50, 6412 (1994). hep-th/9405162

11. J. Sadeghi, M.R. Setare, B. Pourhassan, Acta Phys. Polon. B 40, 251 (2009). arXiv:0707.0420 [hep-th]

12. S. Hyun, W. Kim, J.J. Oh, E.J. Son, JHEP 0704, 057 (2007). hep-th/0702170

13. W.T. Kim, J.J. Oh, J.H. Park, Phys. Rev. D 60, 047501 (1999). hep-th/9902093

14. E.C. Vagenas, Mod. Phys. Lett. A 17, 609 (2002). hep-th/0108147

15. D.A. Easson, JHEP 0302, 037 (2003). hep-th/0210016

16. D. Grumiller, W. Kummer, D.V. Vassilevich, Phys. Rept. 369, 327 (2002). hep-th/0204253

17. A. Strominger, C. Vafa, Phys. Lett. B 379, 99 (1996). hep-th/9601029

18. G. Lopes Cardoso, T. Mohaupt. Phys. Lett. B 435, 277 (1998). hep-th/9806036

19. T. Regge, J.A. Wheeler, Phys. Rev. 108, 1063 (1957)

20. F.J. Zerilli, Phys. Rev. D 2, 2141 (1970)

21. F.J. Zerilli, Phys. Rev. Lett. 24, 737 (1970)

22. K.D. Kokkotas, B.G. Schmidt, Living Rev. Rel. 2, 2 (1999) gr-qc/9909058

23. H.-P. Nollert, Class. Quant. Grav. 16, R159 (1999)

24. R.A. Konoplya, A. Zhidenko, Rev. Mod. Phys. 83, 793 (2011). arXiv:1102.4014 [gr-qc]

25. J.M. Maldacena, Adv. Theor. Math. Phys. 2, 231 (1998). hep-th/9711200

26. G.T. Horowitz, V.E. Hubeny, Phys. Rev. D 62, 024027 (2000). hep-th/9909056

27. J.D. Bekenstein, Lett. Nuovo Cim. 11, 467 (1974)

28. S. Hod, Phys. Rev. Lett. 81, 4293 (1998) gr-qc/9812002

29. G. Kunstatter, Phys. Rev. Lett. 90, 161301 (2003) gr-qc/0212014

30. M. Maggiore, Phys. Rev. Lett. 100, 141301 (2008). arXiv:0711.3145 [gr-qc]

31. C. Corda, Int. J. Mod. Phys. D 21, 1242023 (2012) arXiv:1205.5251 [gr-qc]

32. C. Corda, Eur. Phys. J. C 73, 2665 (2013). arXiv:1210.7747 [gr-qc]

33. C. Corda, S.H. Hendi, R. Katebi, N.O. Schmidt, JHEP 1306, 008 (2013). arXiv:1305.3710 [gr-qc]

34. C. Corda, S.H. Hendi, R. Katebi, N.O. Schmidt, arXiv:1401.2872 [physics.gen-ph]

35. W.T. Kim, J. Lee, Y.-J. Park, Phys. Lett. B 347, 217 (1995). hep-th/9502114

36. S. 'i. Nojiri, S.D. Odintsov, Phys. Rev. D 59, 044003 (1999) hep-th/9806055

37. V.P. Frolov, A. Zelnikov, Phys. Rev. D 63, 125026 (2001). hep-th/0012252

38. R. Becar, S. Lepe, J. Saavedra, Phys. Rev. D 75, 084021 (2007) gr-qc/0701099

39. R. Becar, S. Lepe, J. Saavedra, Int. J. Mod. Phys. A 25, 1713 (2010)

40. A. Lopez-Ortega, Int. J. Mod. Phys. D 18, 1441 (2009). arXiv:0905.0073 [gr-qc]

41. Ramon Becar, P.A. Gonzalez, Y. Vasquez, arXiv:1404.6023 [gr-qc]

42. X.-Z. Li, J.-G. Hao, D.-J. Liu, Phys. Lett. B 507, 312 (2001) gr-qc/0205007

43. M. Abramowitz, A. Stegun, Handbook of Mathematical Functions (Dover publications, New York, 1970)

44. M. Catalan, E. Cisternas, P.A. Gonzalez, Y. Vasquez, arXiv: 1404.3172 [gr-qc]

45. P.A. Gonzalez, Y. Vasquez, arXiv:1404.5371 [gr-qc] 